\title{
A SEMIBOUNDED CLOSED SYMMETRIC OPERATOR WHOSE SQUARE HAS TRIVIAL DOMAIN
}

\author{
PAUL R. CHERNOFF
}

\begin{abstract}
The existence of closed symmetric operators $T$ such that $D\left(T^{2}\right)=(0)$ was shown by Naimark. This paper gives a simple, explicit construction for such operators and shows that $T$ can be semibounded.
\end{abstract}

Naimark [2] showed there are closed symmetric operators $T$ on a Hilbert space $H$ such that the domain of $T^{2}$ contains only 0 . His construction is rather complicated, and the resulting operators are not very explicit. The aim of this note is to give a simple, concrete construction of such operators. Our construction shows that $T$ may be semibounded as well.

We use the machinery of the Cayley transform (see, e.g., [4, p. 502]). Let $M$ and $N$ be closed subspaces of $H$ and let $V$ be an isometry mapping $M$ onto $N$ such that $(V-I) M=D$ is a dense subspace of $H$. Then $V-I$ is automatically one-to-one, and the relation

$$
T=i(V+I)(V-I)^{-1}
$$

defines a closed symmetric operator $T$ with dense domain $D$. Clearly the domain of $T^{2}$ reduces to $(0)$ precisely when the ranges of $V+I$ and $V-I$ have trivial intersection. This amounts to requiring that $M \cap N=(0)$. Our task is to exhibit $M, N$ and $V$ satisfying all these conditions.

We take for our Hilbert space $L^{2}(S)$, where $S$ is the unit circle. For $M$ we take the Hardy class $H^{2}(S)$. $V$ will be multiplication by a suitable function $\Omega(\theta)$ of modulus one, and $N=V M=\Omega \cdot H^{2}$. Everything turns on the choice of $\Omega$, which is guided by considering the following well-known result of Szegö [3, 1, p. 53]: If $f$ is a nonzero function in $H^{2}$, then

$$
\int_{0}^{2 \pi} \log |f(\theta)| d \theta>-\infty
$$

In particular, $f$ cannot vanish on a set of positive measure.

Define $\Omega(\theta)=\exp \left(i e^{-1 / \theta}\right)$ for $0<\theta<\pi, \Omega(\theta)=-1$ for $\pi \leqslant \theta \leqslant 2 \pi$. The salient properties of $\Omega$ are the following. First, $\Omega(\theta)=-1$ on a set of positive measure; second, $\Omega(\theta) \neq 1$, but

$$
\int_{0}^{2 \pi} \log |\Omega(\theta)-1| d \theta=-\infty
$$

third, the imaginary part of $\Omega$ is nonnegative.

Received by the editors January 27, 1983.

1980 Mathematics Subject Classification. Primary 47B25. 
The first property guarantees that $M \cap N=(0)$. Indeed, suppose $f \in H^{2}$ is such that $\Omega \cdot f$ is also in $H^{2}$, so that $\Omega \cdot f \in M \cap N$. Then $(\Omega+1) f \in H^{2}$ but vanishes on a set of positive measure. Hence $(\Omega+1) f=0$, which implies $f$ is supported on the arc $\pi \leqslant \theta \leqslant 2 \pi$, hence $f=0$.

The second property guarantees that $(V-I) M=(\Omega-1) \cdot H^{2}$ is dense. For if $g \in L^{2}$ is orthogonal to $(\Omega-1) \cdot H^{2}$, it follows that $(\Omega-1) \bar{g}=f$ belongs to $H^{2}$. But (3) implies $\int \log |f| \mathrm{d} \theta=-\infty$, so by Szegö's theorem $f$ vanishes, hence $g$ vanishes.

It follows that the operator $T$ defined by (1) does what we want. Note that $T$ is given by multiplication by $F=i(\Omega+1)(\Omega-1)^{-1}$ on the domain $D=(\Omega-1) \cdot H^{2}$. Moreover the function $F$ is nonnegative because $\operatorname{Im}(\Omega) \geqslant 0$. Hence the closed symmetric operator $T$ is nonnegative.

\section{REFERENCES}

1. K. Hoffman, Banach spaces of analytic functions, Prentice-Hall, Englewood Cliffs, N.J., 1962.

2. M. Naimark, On the square of a closed symmetric operator, Dokl. Akad. Nauk SSSR 26 (1940), 866-870; ibid. 28 (1940), 207-208.

3. G. Szegö, Über die Randwerte einer analytischen Funktion, Math. Ann. 84 (1921), 232-244.

4. K. Yosida, Functional analysis, 3rd ed., Springer-Verlag, Berlin and New York, 1971.

Department of Mathematics, University of California, Berkeley, California 94720 